\title{
Testes de degradação por foto deposição de um refletor solar óptico para controle térmico de satélites
}

\author{
Degradation tests by photo-deposition for an optical solar reflector for using \\ in satellite thermal control
}

Marcos Galante Boato ${ }^{1}$, Ezio Castejon Garcia, Marcio Bueno dos Santos², George Favale e Fernandes²

\section{RESUMO}

Em um ambiente de alto-vácuo, alguns materiais que compõe o satélite "degaseificam". Tais vapores ou contaminantes acabam depositando-se nas superfícies de controle térmico que somados a ação da radiação ultravioleta, escurecem e modificam as propriedades termo-ópticas. O objetivo desta pesquisa foi verificar experimentalmente a ação da radiação ultravioleta nos aspectos físicos do escurecimento das superfícies do Optical Solar Reflector (OSR), utilizados como ferramenta para controle térmico de satélites. Além disso, o estudo a relação entre a temperatura da superfície do OSR e a permanência da camada de contaminante depositada.

Palavras-chave: Fotoquímico, Degradação, Refletor solar óptico.

\begin{abstract}
In a high-vacuum environment, some materials of the satellite outgas. Theses vapours or contaminants deposite theirselves on the thermal control surfaces, and with the action of the ultraviolet radiation, they get darkness and modify the thermooptical properties of the surfaces. The objective of this research was to verify experimentally the action of the ultraviolet radiation in the physical aspects of darkness of Optical Solar Reflector's (OSR) surface, used as a tool for satellites' thermal control. In addition, the study verified the relation between the OSR's surface temperature and the permanence of the contamination's coating deposited.
\end{abstract}

Keywords: Photochemical, Degradation, Optical solar reflector. 


\section{INTRODUÇÃO}

O Refletor Solar Óptico (OSR) é basicamente um espelho de segunda superfície (uma lâmina de quartzo ou teflon com uma fina camada de prata depositada no seu verso $)^{(2,4)}$. São dispositivos utilizados em determinadas partes do satélite para a rejeição de calor para o meio espacial. Na maioria das vezes este radiador é composto por uma lâmina de borossilicato ou sílica fundida, retangular ou quadrada.

O OSR fabricado com lamínula de sílica fundida normalmente não degrada suas propriedades de absortividade solar $\left(\alpha_{\mathrm{s}}\right)$ e emissividade infravermelha $(\varepsilon)$ no ataque de partículas de alta energia, radiação ultravioleta e outros elementos presentes no ambiente espacial. Porém, a degradação ocorre oriunda dos contaminantes provindos da "degaseificação" de orgânicos voláteis que são exauridos do satélite ou do foguete no estágio de posicionamento do satélite $e^{(2,3,5)}$.

O mecanismo de degradação ainda não é bem conhecido, porém está comprovado que esse efeito causa o aumento de absortividade solar nestes radiadores tipo espelho. O que se conhece é que os orgânicos voláteis, oriundos de "degaseificação", se depositam na superfície do espelho. Esses contaminantes orgânicos, por efeito fotoquímico, acabam aderindo na superfície dos espelhos e assim deixando-os enegrecidos. O efeito do enegrecimento aumenta a opacidade, do vidro, para a banda da luz visível, fazendo com que ocorra o aumento na absortividade solar ${ }^{(5)}$.

Estudos observaram que quanto mais a superfície estiver quente, menor será a taxa de deposição de orgânicos voláteis ${ }^{1,5}$, amenizando assim a degradação. A Fig. 1 apresenta a espessura, em angstroms (Å), da camada contaminante depositada em relação à temperatura da superfície do satélite.

A fim de verificar o efeito da degradação devido ao processo fotoquímico de orgânicos voláteis depositados nas superfícies do satélite, foi elaborado um simples experimento para comprovar, in sito, o fenômeno demonstrado na Fig. 1. Para a simulação espacial prevista para o voo de um satélite foram utilizadas quatro lamínulas de OSR, sendo três delas previamente contaminadas com um agente orgânico antes do teste; estas foram individualmente aquecidas com um sistema independente de aquecedores elétricos



Figura 1: Espessura de filme de contaminante depositado versus temperatura da superfície do satélite (modificada de Stewart et al. 1990) e expostas à luz ultravioleta em vácuo. Antes do teste de degradação foi escolhida arbitrariamente a temperatura de cada lamínula contaminada. A Tabela 1 mostra a temperatura imposta a cada OSR durante o experimento.

Tabela 1: Temperatura em cada OSR.

\begin{tabular}{|c|c|}
\hline Condição para cada OSR & Temperatura imposta em ${ }^{\circ} \mathbf{C}$ \\
\hline $1^{\circ}$ OSR contaminado & $80 \pm 05$ \\
\hline $2^{\circ}$ OSR contaminado & $50 \pm 05$ \\
$3^{\circ}$ OSR contaminado & $25 \pm 05$ \\
$4^{\circ}$ OSR não contaminado & $25 \pm 05$ \\
\hline
\end{tabular}

O objetivo desse experimento foi verificar duas características:

1. A influência do aumento da temperatura na diminuição da quantidade de contaminante na superfície do OSR;

2. E a influência da exposição a radiação ultravioleta tipo $\mathrm{C}$ na mudança da absortividade solar $\left(\alpha_{s}\right)$ no OSR devido à reação fotoquímica do contaminante.

\section{APARATO EXPERIMENTAL}

Para reproduzir o efeito da degradação dos OSR's em solo (simular o ambiente espacial) foi montado o experimento dentro de uma câmara de alto-vácuo com uma lâmpada de ultravioleta tipo C e um sistema de monitoramento da temperatura dos OSR's. Detalhadamente, foram utilizados os seguintes equipamentos e materiais para compor o aparato experimental:

- Uma câmara de alto-vácuo com sistema de bombeamento primário mecânico seco e bombeamento secundário composto por bomba turbo molecular, no Laboratório Associado de Plasma do INPE;

- Dois aquecedores de película, conhecidos por "skin heaters";

- Duas fontes de tensão da marca LAMBDA modelo GEN150-5;

- Seis lâmpadas de vapor de mercúrio de baixa pressão da marca OSRAM com cada uma contendo $2,8 \mathrm{~W}$ de potência luminosa na faixa de 200 à $280 \mathrm{~nm}$;

- Três termopares tipo "T";

- Equipamento de leituras dos termopares da marca Minipa;

- Porta amostra feito com chapas de alumínio com espessura de $2 \mathrm{~mm}$;

- Óleo de bomba difusora da marca Dow Corning DC-704 (componente químico tetrametiltetrafeniltrisiloxane).

No experimento, foram utilizadas quatro amostras de OSR's, sendo duas delas fixadas em um porta-amostra, e as outras duas fixadas separadamente em porta-amostras individuais. 
Também foram instalados os "skin heaters" e termopares nos porta-amostras que encontravam-se com apenas um OSR; no terceiro porta-amostras que encontravam-se duas lamínulas juntas apenas foi instalado o terceiro termopar. No término da instrumentação das lamínulas, três delas (duas com o sistema de aquecimento acoplado e a terceira sem aquecimento) foram contaminadas com uma fina camada de tetrametiltetrafeniltrisiloxane (DC-704), como apresentado na Fig. 2. O componente encontrado na contaminação das superfícies do satélite é compatível ao óleo DC- $704^{(5)}$.

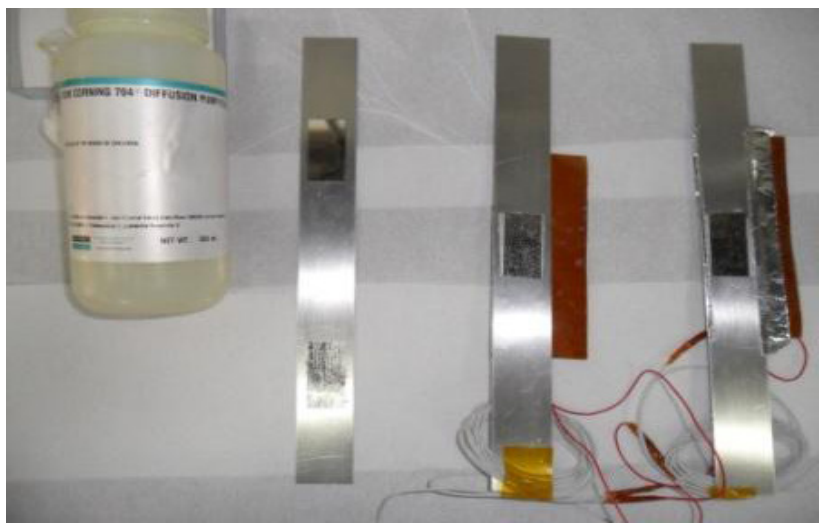

Figura 2: Amostras fixadas e contaminadas com o DC-704.

A fina camada de óleo depositada sobre as amostras foi feita utilizando-se um lenço especial para limpeza de instrumentos para vácuo, que não deixa fiapos ou vestígios, embebido com o óleo DC-704, o contaminante, e aplicado diretamente sobre a superfície dos OSR's, respeitando a direção da aplicação, a fim de buscar uniformidade na película.
Os "skin heaters" e os termopares das amostras foram acoplados no porta-amostras e os conjuntos posicionados dentro da câmara de vácuo. As conexões elétricas com o meio externo foram feitas por conectores herméticos de tensão para interface de vácuo-ambiente externo, conhecidos como feedthroughs.

A fonte de radiação ultravioleta, composta por seis lâmpadas de vapor de mercúrio, foi instalada sobre as amostras em uma janela no topo da câmara. Essa janela, fabricada em sílica fundida, permite a passagem de $98 \%$ de toda radiação ultravioleta emitida pelo conjunto de lâmpadas e incidida sobre as amostras.

Cada aquecedor foi alimentado por uma fonte de tensão LAMBDA controlada manualmente de acordo com a leitura da temperatura. A Fig. 3 mostra o esquema do arranjo experimental composto por lâmpadas, amostras, as ligações dos instrumentos de medida e alimentação.

\section{RESULTADOS E DISCUSSÕES.}

Antes de ser iniciado o teste de degradação, foi medida a absortividade térmica na banda solar $\left(\alpha_{\mathrm{s}}\right)$ daquelas lamínulas com a contaminação e sem a contaminação (grupo controle). Para o experimento de degradação, foi seguida uma sequência de passos para simular o que ocorreria no ambiente espacial:

1. Ligar o sistema de bombeamento primário e secundário de vácuo: pressão final de $1,16 \times 10^{-5} \mathrm{mbar}$;

2. Ligar as fontes de tensão de alimentação dos "skin heaters" e reguladas potências para que as temperaturas das amostras chegassem próximo ao determinado;

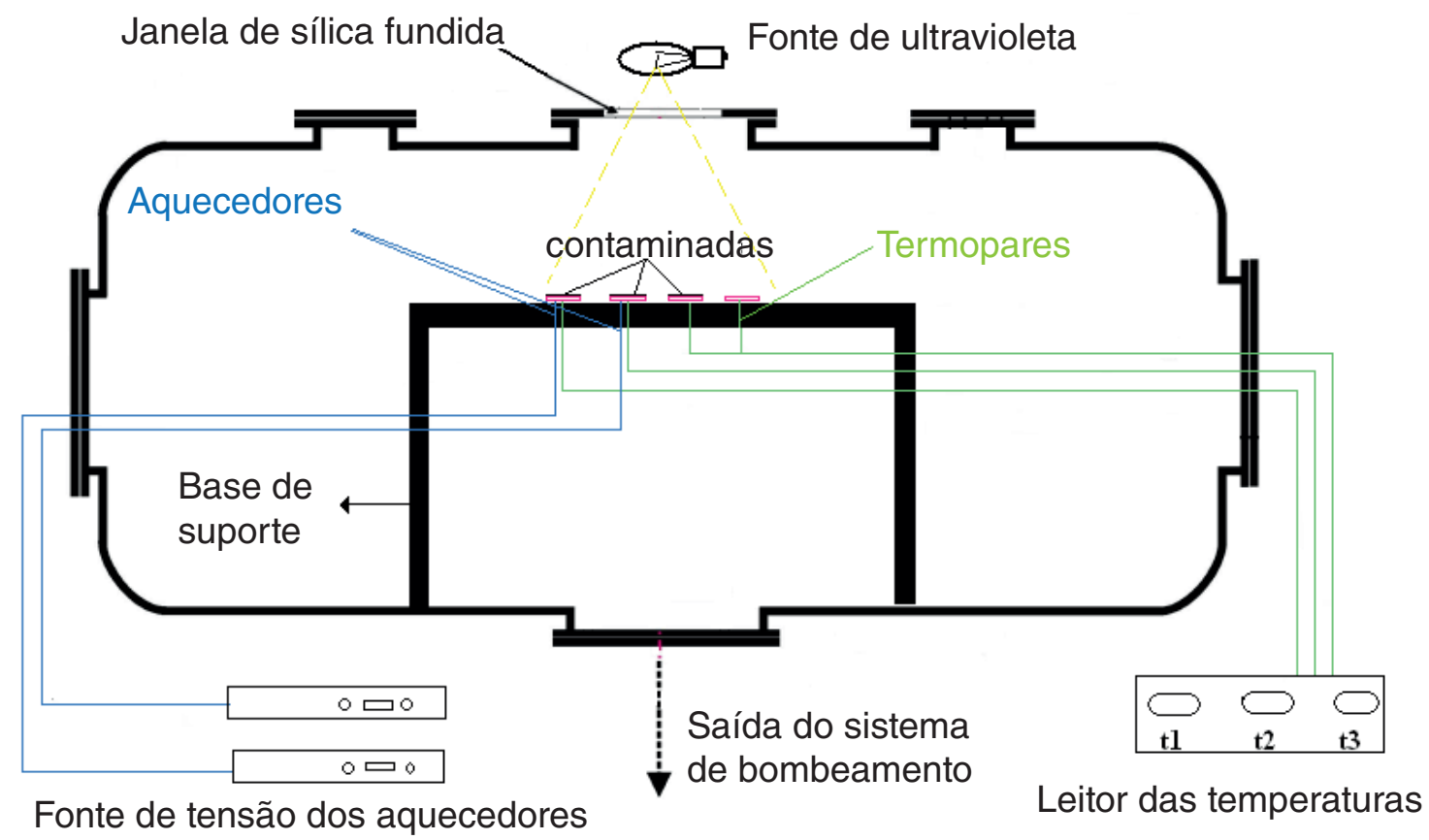

Figura 3: Esquema do aparato experimental realizado. 
3. Ligar a fonte de radiação ultravioleta e mantida a temperatura dos espécimes estabilizada até o final do teste.

O teste de degradação teve a duração total de 11 horas de exposição a radiação ultravioleta, em alto vácuo e aquecimento constante e estável das amostras. A lâmpada ultravioleta, na faixa de 200 a $280 \mathrm{~nm}$, teve sua potência luminosa total de 16,8 Watts, o que forneceu radiação equivalente à aproximadamente 38 horas de radiação solar, na mesma faixa de comprimentos de onda. A Fig. 4 apresenta os porta-amostras recebendo a radiação ultravioleta pela janela superior da câmara de vácuo.

Ao final do teste, foram retirados os porta-amostras de dentro da câmara e foi realizado um exame visual. $\mathrm{O}$ que pôde ser observado foi que a lamínula que ficou com a temperatura de aproximadamente $80^{\circ} \mathrm{C}$ encontrava-se, aparentemente, com quantidade menor de contaminante que a exposta a aproximadamente a $25^{\circ} \mathrm{C}$ (Fig. 5). Isso sugere que as superfícies com temperaturas mais elevadas têm menores taxas de deposição porque, ao se depositarem elas rapidamente evaporam.

Além disso, os $\alpha_{s}$ das amostras foram novamente medidos. Os resultados mostraram leve mudança em comparação com as medidas feitas antes do experimento de degradação. A Tabela 2 mostra os dados no início e no final do teste.

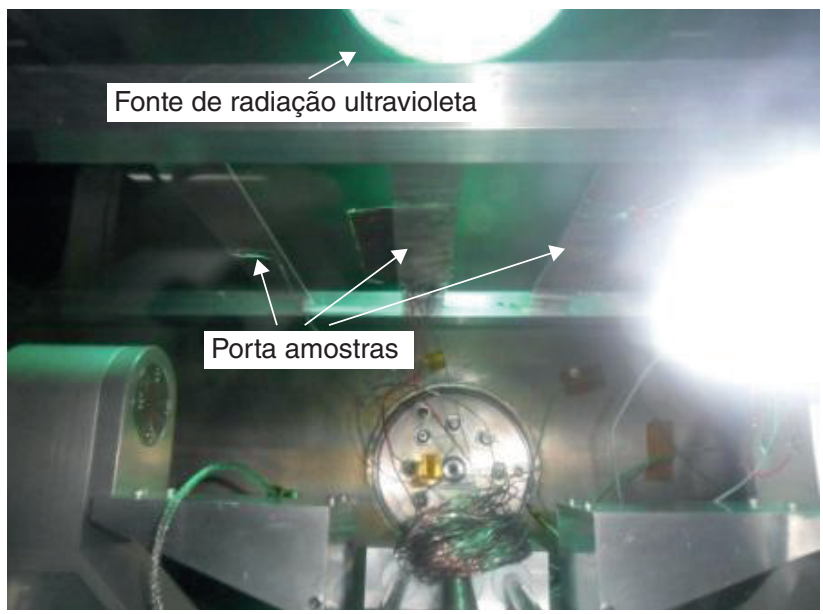

Figura 4: Lamínulas recebendo a radiação emitida pelo conjunto de lâmpadas ultravioleta.

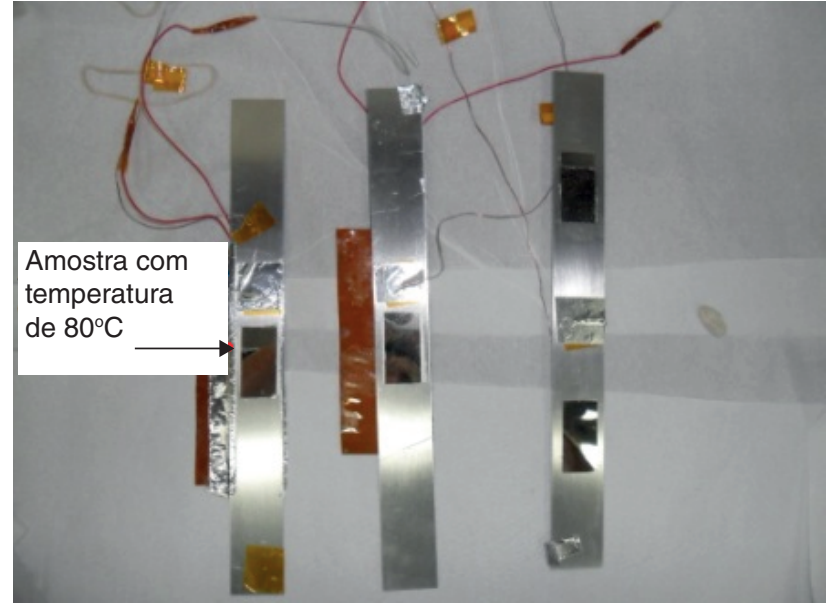

Figura 5: Amostras ao final do experimento.

As medidas de $\alpha_{\mathrm{s}}$ no início e no final do teste de degradação não mostraram variações expressivas como esperado, provavelmente porque o tempo de exposição a radiação ultravioleta, em vácuo, foi inferior se comparado com a exposição prevista para voo de um satélite exposto em ambiente espacial.

\section{CONCLUSÕES}

O depósito de contaminante no experimento sobre o OSR foi maior se comparado com a literatura para situações reais em ambiente espacial. O tempo de exposição foi insuficiente para ocorrer um enegrecimento que pudesse ser mais significativo, alterando claramente as medidas de $\alpha_{s}$.

O que pode ser observado neste experimento é:

- O teste valida a relação hipotética entre temperatura de superfície do OSR e taxa de contaminantes depositados sobre ele;

- A ocorrência da degradação fotoquímica do orgânico volátil, oriundo do satélite, na presença da radiação ultravioleta em vácuo, é um processo lento e necessita de um período significativamente maior de exposição a essa radiação.

Tabela 2: Dados do teste de degradação com relação à absortividade térmica solar $\left(\alpha_{\mathrm{s}}\right)$.

\begin{tabular}{|c|c|c|c|c|}
\hline Dados retirados do teste & $\begin{array}{c}\text { Medidas iniciais } \\
\text { de } \alpha_{s}\end{array}$ & Início dos testes & Final dos testes & Medidas finais de $\alpha_{s}$ \\
\hline $1^{\circ}$ OSR contaminada & $0,020 \pm 0,004$ & - & - & $0,022 \pm 0,004$ \\
\hline $2^{\circ}$ OSR contaminada & $0,018 \pm 0,002$ & - & - & $0,020 \pm 0,002$ \\
\hline $3^{\circ} \mathrm{OSR}$ contaminada & $0,020 \pm 0,001$ & - & - & $0,022 \pm 0,001$ \\
\hline $4^{\circ}$ OSR não contaminada & $0,020 \pm 0,003$ & - & - & $0,020 \pm 0,003$ \\
\hline Pressão da câmara & - & $1.16 \times 10^{-5} \mathrm{mbar}$ & $1.15 \times 10^{-7} \mathrm{mbar}$ & - \\
\hline Temperatura $1^{\circ}$ OSR & - & $80,9^{\circ} \mathrm{C}$ & $78,6^{\circ} \mathrm{C}$ & - \\
\hline Temperatura $2^{\circ}$ OSR & - & $46,4^{\circ} \mathrm{C}$ & $47.8^{\circ} \mathrm{C}$ & - \\
\hline Temperatura $3^{\circ}$ OSR & - & $24,1^{\circ} \mathrm{C}$ & $32,3^{\circ} \mathrm{C}$ & - \\
\hline Temperatura $4^{\circ}$ OSR & - & $24,1^{\circ} \mathrm{C}$ & $32,3^{\circ} \mathrm{C}$ & - \\
\hline
\end{tabular}




\section{REFERÊNCIAS}

1. DEVER, J.A., BRUCKNER, E.J., SCHEIMAN, D.A., STIDHAM, C.R., Contamination and Space Environmental Effects on Solar Cells and Thermal Control Surfaces. Journal of Spacecraft and Rocket. Vol. 32, n 5, Set.-Out., 1995. Pag. 850.

2. GILMORE, D.G., Satellite Thermal Control Handbook. Volume I: Fundamental Technologies, Second Edition, The Aerospace Corporation Press, El Segundo, California, 1994.

3. HELTZEL, S., SEMPRIMOSCHNING, C.O.A., VAN EESBEEK, M.R.J., Environmental Testing of Thermal Control Materials at Elevated Temperature and Intense Ultraviolet Radiation.
Journal of Spacecraft and Rocket. Vol. 46, n² 2, Mar.-Abr. 2009. $248 \mathrm{p}$.

4. MARSHALL, K.N., BREUCH, R.A. Optical Solar Reflector: A highly stable, low $\alpha_{s} / \varepsilon$ Spacecraft Thermal Control Surface. Journal of Spacecraft and Rocket. Vol. 5, n 9, Set., 1968. 1051 pp.

5. STEWART, T. B., ARNOLD, G. S., HALL, D. E., MARVIN, D. C., HWANG, W. C., YOUNGOWL, R. C., MARTEN, H. D., Photochemical Spacecraft Self-Contamination: Laboratory Results and Systems Impacts. Laboratory Operation, The Aerospace Corporation, El Segundo, 25, Jul. 1990 